\title{
SOME PROPERTIES OF GREEN'S FUNCTIONS OF SHILOV-TYPE PARABOLIC SYSTEMS
}

\author{
V. LITOVCHENKO AND G. UNGURYAN
}

Received 15 September, 2016

\begin{abstract}
For Shilov-type parabolic systems with nonnegative genus and coefficients of bounded smoothness, the properties of differentiability of Green's function with respect to spatial variables are studied.
\end{abstract}

2010 Mathematics Subject Classification: 35K40; 35A08

Keywords: Cauchy problem, Shilov-type parabolic systems, Green's function

\section{INTRODUCTION}

Developing the idea advanced by Ya.I. Zhitomirskii in [10] concerning the description of the systems of partial differential equations parabolically steady to a change in coefficients, the following wide class of parabolic systems with variable coefficients was defined in [7]:

$$
\partial_{t} u(t ; x)=\left\{P_{0}\left(t ; i \partial_{x}\right)+P_{1}\left(t, x ; i \partial_{x}\right)\right\} u(t ; x), \quad(t ; x) \in \Pi_{(0 ; T]} .
$$

Here, $u:=\operatorname{col}\left(u_{1}, \ldots, u_{m}\right), \Pi_{(0 ; T]}:=\left\{(t ; x): t \in(0 ; T], x \in \mathbb{R}^{n}\right\}$, and $P_{0}\left(t ; i \partial_{x}\right)$ and $P_{1}\left(t, x ; i \partial_{x}\right)$ are matrix differentiable expressions of the orders, respectively, $p$ and $p_{1}, p>p_{1} \geq 0$, with coefficients depending on the time variable $t$. In this case, the coefficients of the expression $P_{1}$ can depend also on the spatial variable $x$. It is also assumed that the corresponding system

$$
\partial_{t} u(t ; x)=P_{0}\left(t ; i \partial_{x}\right) u(t ; x), \quad(t ; x) \in \Pi_{(0 ; T]},
$$

is uniformly parabolic by Shilov with parabolicity index $h, 0<h \leq p$, nonnegative genus $\mu$, and reduced order $p_{0}$ [2]. In other words, there exist constants $\delta_{0}>0$ and $\delta_{1} \geq 0$ such that, for all $x \in \mathbb{R}^{n}$ and $t \in[0 ; T]$, the following inequality holds:

$$
\Lambda(t ; x):=\max _{j \in \mathbb{N}_{m}} \operatorname{Re} \lambda_{j}(t ; x) \leq-\delta_{0}\|x\|^{h}+\delta_{1} .
$$

Here, $\lambda_{j}$ are roots of the equation $\operatorname{det}\left(P_{0}(t ; \zeta)-\lambda E\right)=0, \zeta \in \mathbb{C}^{n}, E$ is the identity matrix of the order $m,\|\cdot\|$ is the Euclid norm in $\mathbb{R}^{n}$, and $\mathbb{N}_{m}:=\{1,2, \ldots, m\}$.

We recall also that the reduced order $p_{0}$ of a system is called the exact power order of growth of a function $\Lambda(\cdot ; \cdot)$ in the complex space $\mathbb{C}^{n}$ (for a parabolic system, 
we have always $\left.p_{0}>1\right)$. In addition, the genus of a system parabolic by Shilov is called the largest index $\mu$ such that the function $\Lambda(\cdot ; \cdot)$ in the domain $G^{\mu}(K):=$ $[0 ; T] \times\left\{x+i y \in \mathbb{C}^{n}:\|y\| \leq K(1+\|x\|)^{\mu}\right\}$ with some $K>0$ satisfies the estimate

$$
\Lambda(t ; x+i y) \leq-\delta_{*}\|x\|^{h}+\delta_{1}, \delta_{*}>0, \delta_{1} \geq 0 .
$$

It is known that $1-\left(p_{0}-h\right) \leq \mu \leq 1$.

The following condition must be satisfied for (1.1):

$$
\text { (A) : } 0 \leq p_{1}<h-n\left(1-h \mu / p_{0}\right)-(m-1)(p-h) \text {. }
$$

It is obvious that the class of systems (1.1) envelops completely the class of Shilovtype parabolic systems with nonnegative genus. In this connection, it was proposed in [10] to call such systems Shilov-type parabolic systems with nonnegative genus and variable coefficients. To a certain extent, these systems solve the problem of proper extension of the notion of parabolicity by Shilov to the linear systems of equations with variable coefficients, since the Shilov class of systems is not parabolically stable to a change in its coefficients [4].

Let us consider systems (1.1) under the condition that their coefficients are the complex-valued functions that are continuous in the variable $t$, infinitely differentiable with respect to the variable $x$, and bounded together with their derivatives in a ball $\Pi_{[0 ; T]}$. For these systems, Green's function $Z(t, x ; \tau, \xi), 0 \leq \tau<t \leq T$, $\{x, \xi\} \subset \mathbb{R}^{n}$, was constructed, and its main properties such as the smoothness and the behavior in vicinities of infinitely remote spatial points were studied [7]. On the basis of these results, the theory of the Cauchy problem was developed in $[5,8,9]$ for such systems in spaces of the type $S$ by Gelfand and Shilov [3]. In particular, the proper solvability of the Cauchy problem with generalized initial data like the Gevrey ultradistributions was established, the form of a representation of classical solutions with generalized limiting values on the input hyperplane was obtained, their qualitative properties were studied, and the sets of generalized input functions, for which the corresponding solutions are elements of the Schwartz space $S$ or some space of the type $S$, were described.

In order to get similar results under weaker conditions imposed on the coefficients of system (1.1), it is necessary firstly to clarify the properties of corresponding Green's function $Z$. In the present work, we will study properties of the function $Z(t, x ; \tau, \xi)$ with respect to spatial variables $x$ and $\xi$ under the minimum conditions of smoothness imposed on the coefficients of this system.

\section{AUXILIARY INFORMATION}

Let the differential expressions $P_{0}$ and $P_{1}$ of system (1.1) have the structure

$$
P_{0}\left(t ; i \partial_{x}\right)=\sum_{|k|_{+} \leq p} A_{0, k}(t) \partial_{x}^{k}, P_{1}\left(t, x ; i \partial_{x}\right)=\sum_{|k|_{+} \leq p_{1}} A_{1, k}(t ; x) \partial_{x}^{k},
$$


where $A_{0, k}(t):=i^{|k|_{+}}\left(a_{0, k}^{l j}(t)\right)_{l, j=1}^{m}, A_{1, k}(t ; x):=i^{|k|_{+}}\left(a_{1, k}^{l j}(t ; x)\right)_{l, j=1}^{m}$ are matrix coefficients, $i$ is the imaginary unity, and $|k|_{+}:=k_{1}+\ldots+k_{n}, k \in \mathbb{Z}_{+}^{n}$.

By $G(t, \tau ; \cdot), 0 \leq \tau<t \leq T$, we denote Green's function of system (1.2). It is known that $G(t, \tau ; \cdot)=F\left[\Theta_{\tau}^{t}(\xi)\right](t, \tau ; \cdot)$, where $F[\cdot]$ is the Fourier transformation operator, and $\Theta_{\tau}^{t}(\cdot)$ is a matriciant of the corresponding Fourier duality of the system. The following proposition is proper [5,6]:

$\forall T>0 \exists \delta>0 \forall k \in \mathbb{Z}_{+}^{n} \exists c>0 \forall t \in(\tau ; T] \forall \tau \in[0 ; T) \forall\{x, \xi\} \subset \mathbb{R}^{n}:$

$$
\left|\partial_{x}^{k} G(t, \tau ; x-\xi)\right| \leq c(t-\tau)^{-\frac{n+|k|_{+}+\gamma}{h}} e^{-\delta\left(\frac{\|x-\xi\|}{(t-\tau)^{\alpha}}\right)^{\frac{1}{1-\alpha}}},
$$

where $\gamma:=(m-1)(p-h)$ and $\alpha:=\mu / p_{0},\left|\left(a_{l j}\right)_{l, j=1}^{m}\right|:=\max _{\{l, j\} \subset \mathbb{N}_{m}}\left|a_{l j}\right|$.

Here, we consider systems (1.1), which satisfy, in addition to condition (A), the following condition:

(B): the coefficients $a_{0, k}^{l j}(t), a_{1, k}^{l j}(t ; x)$ are continuous in the variable $t$ uniformly with respect to $x$, differentiable with respect to the variable $x$ up to the order $\alpha_{*}$ inclusively, and bounded together with their derivatives by complex-valued functions in a ball $\Pi_{[0 ; T]}$.

In [7], Green's function of system (1.1) was constructed in the form

$$
Z(t, x ; \tau, \xi)=G(t, \tau ; x-\xi)+W(t, x ; \tau, \xi),(t, x ; \tau, \xi) \in \Pi_{T}^{2},
$$

where $\Pi_{T}^{2}:=\left\{(t, x ; \tau, \xi) \mid 0 \leq \tau<t \leq T,\{x, \xi\} \subset \mathbb{R}^{n}\right\}$,

$$
W(t, x ; \tau, \xi):=\int_{\tau}^{t} d \beta \int_{\mathbb{R}^{n}} G(t, \beta ; x-y) \Phi(\beta, y ; \tau, \xi) d y,
$$

and

$$
\Phi(t, x ; \tau ; \xi)=\sum_{l=1}^{\infty} K_{l}(t, x ; \tau, \xi)
$$

where

$$
\begin{gathered}
K_{1}(t, x ; \tau, \xi):=P_{1}\left(t, x ; i \partial_{x}\right) G(\tau, \xi ; x-\xi), \\
K_{l}(t, x ; \tau, \xi):=\int_{\tau}^{t} d \beta \int_{\mathbb{R}^{n}} K_{1}(t, x ; \beta, y) K_{l-1}(\beta, y ; \tau, \xi) d y, l>1 .
\end{gathered}
$$

In this case, it was established that condition (A) and the boundedness of the coefficients of system (1.1) ensure the absolute uniform convergence of the functional series (2.4) for all $\{x, \xi\} \subset \mathbb{R}^{n}, t \in(\tau ; T]$, and $\tau \in[0, T)$. Moreover, its sum $\Phi$ and the iterated kernels $K_{l}$ satisfy the estimates

$$
|\Phi(t, x ; \tau, \xi)| \leq c_{1}(t-\tau)^{\alpha_{0}-(1+\alpha n)} e^{-\delta_{1}\left(\frac{\|x-\xi\|}{(t-\tau)^{\alpha}}\right)^{\frac{1}{1-\alpha}}},
$$




$$
\begin{aligned}
\left|K_{l}(t, x ; \tau, \xi)\right| \leq & c_{0}^{l}\left(\prod_{j=1}^{l-1} c_{(j \varepsilon)} B\left(\alpha_{0}, j \alpha_{0}\right)\right) \\
& \times(t-\tau)^{l \alpha_{0}-(1+\alpha n)} e^{-\delta(1-(l-1) \varepsilon)\left(\frac{\|x-\xi\|}{(t-\tau)^{\alpha}}\right)^{\frac{1}{1-\alpha}}}, \varepsilon \in(0 ; 1),
\end{aligned}
$$

with the estimating constants independent of $t, \tau, x$, and $\xi$. Here $\alpha_{0}:=1+\alpha n-(n+$ $\left.p_{1}+\gamma\right) / h>0$ and $B(\cdot, \cdot)$ is the Euler beta-function.

We note that estimates (2.1) and (2.6) for $\{x, \xi\} \subset \mathbb{R}^{n}$ and $0 \leq \tau<t \leq T$ guarantee the absolute convergence of the integral, by which the potential $W$ is determined. Thus, the matrix function $Z(t, x ; \tau, \xi)$ is properly determined by formula (2.2) on the whole set $\Pi_{T}^{2}$.

Completing this item, we present the following estimates from [1], which will be of importance in what follows:

$$
\begin{aligned}
& e^{-\delta\left\{\left(\frac{\|x-y\|}{(t-\beta)^{\alpha}}\right)^{\frac{1}{1-\alpha}}+\left(\frac{\|y-\xi\|}{(\beta-\tau)^{\alpha}}\right)^{\frac{1}{1-\alpha}}\right\}} \leq e^{-\delta\left(\frac{\|x-\xi\|}{(t-\tau)^{\alpha}}\right)^{\frac{1}{1-\alpha}}} \\
& \int_{\mathbb{R}^{n}} e^{-\delta\left\{\left(\frac{\|x-y\|}{(t-\beta)^{\alpha}}\right)^{\frac{1}{1-\alpha}}+\left(\frac{\|y-\xi\|}{(\beta-\tau)^{\alpha}}\right)^{\frac{1}{1-\alpha}}\right\}} \frac{d y}{((t-\beta)(\beta-\tau))^{\alpha n}} \\
& \leq \frac{c_{\varepsilon} e^{-\delta(1-\varepsilon)\left(\frac{\|x-\xi\|}{(t-\tau)^{\alpha}}\right)^{\frac{1}{1-\alpha}}}, \delta>0}{(t-\tau)^{\alpha n}}, \delta
\end{aligned}
$$

(here, $\{x, y, \xi\} \subset \mathbb{R}^{n}, \beta \in(\tau ; t), 0 \leq \tau<t \leq T, \varepsilon \in(0 ; 1)$, and $\delta>0$, and the quantity $c_{\varepsilon}$ depends only on $\varepsilon$ ).

\section{Properties of Green's Function}

First, we estimate the derivatives of the iterated kernels $K_{l}$.

According to representation (2.5), the smoothness of the kernel $K_{1}(t, x ; \tau, \xi)$ in the spatial variables $x$ and $\xi$ is determined, respectively, by the smoothness of the coefficients of system (1.1) and the function $G(t, \tau ; x-\xi)$. Therefore, there exist the derivatives $\partial_{\xi}^{r} \partial_{x}^{q} K_{1}$ for $\{r, q\} \subset \mathbb{Z}_{+}^{n},|q|_{+} \leq \alpha_{*}$, and the following equality holds:

$$
\partial_{\xi}^{r} \partial_{x}^{q} K_{1}(t, x ; \tau, \xi)=\sum_{|k|_{+} \leq p_{1}} \sum_{l=0}^{q} C_{q}^{l}\left(\partial_{x}^{l} A_{1, k}(t ; x)\right)\left(\partial_{(x-\xi)}^{k+r+q-l} G(t, \tau ; x-\xi)\right),
$$

where $C_{q}^{l}$ is a binomial coefficient. From whence, with regard for condition (B) and estimate (2.1) for $\{r, q\} \subset \mathbb{Z}_{+}^{n},|q|_{+} \leq \alpha_{*},(t, x ; \tau, \xi) \in \Pi_{T}^{2}$, we get

$$
\left|\partial_{\xi}^{r} \partial_{x}^{q} K_{1}(t, x ; \tau, \xi)\right| \leq c_{r, q}(t-\tau)^{-\frac{n+p_{1}+\gamma+|r+q|_{+}}{h}} e^{-\delta\left(\frac{\|x-\xi\|}{(t-\tau)^{\alpha}}\right)^{\frac{1}{1-\alpha}}}
$$


(here, the estimating constants are independent of $t, \tau, x$, and $\xi$ ).

For $l>1$, we will use the representation

$$
\begin{aligned}
K_{l}(t, x ; \tau, \xi)= & \int_{\tau}^{t_{1}} d \beta \int_{\mathbb{R}^{n}} K_{1}(t, x ; \beta, \eta+\xi) K_{l-1}(\beta, \eta+\xi ; \tau, \xi) d \eta \\
& +\int_{t_{1}}^{t} d \beta \int_{\mathbb{R}^{n}} K_{1}(t, x ; \beta, x-z) K_{l-1}(\beta, x-z ; \tau, \xi) d z, t_{1}:=\frac{t+\tau}{2} .
\end{aligned}
$$

According to it,

$$
\begin{aligned}
& \partial_{\xi}^{r} \partial_{x}^{q} K_{l}(t, x ; \tau, \xi)=\sum_{\left|r_{1}\right|_{+} \leq|r|_{+}} C_{r}^{r_{1}} \int_{\tau}^{t_{1}} d \beta \int_{\mathbb{R}^{n}}\left(\partial_{\xi}^{r_{1}} \partial_{x}^{q} K_{1}(t, x ; \beta, \eta+\xi)\right) \\
& \times\left(\partial_{\xi}^{r-r_{1}} K_{l-1}(\beta, \eta+\xi ; \tau, \xi)\right) d \eta+\sum_{\left|q_{1}\right|_{+} \leq|q|_{+}} C_{q}^{q_{1}} \int_{t_{1}}^{t} d \beta \int_{\mathbb{R}^{n}}\left(\partial_{x}^{q_{1}} K_{1}(t, x ; \beta, x-z)\right) \\
& \times\left(\partial_{\xi}^{r} \partial_{x}^{q-q_{1}} K_{l-1}(\beta, x-z ; \tau, \xi)\right) d z,|q|_{+} \leq \alpha_{*},(t, x ; \tau, \xi) \in \Pi_{T}^{2} .
\end{aligned}
$$

Hence, the estimation of $\left|\partial_{\xi}^{r} \partial_{x}^{q} K_{l}(t, x ; \tau, \xi)\right|$ is reduced to that of the expressions

$$
\begin{gathered}
\left|\partial_{\xi}^{r} \partial_{x}^{q} K_{1}(t, x ; \tau, \eta+\xi)\right|, \quad\left|\partial_{x}^{q} K_{1}(t, x ; \tau, x-z)\right|, \\
\left|\partial_{\xi}^{r} K_{l-1}(t, \eta+\xi ; \tau, \xi)\right|, \quad\left|\partial_{\xi}^{r} \partial_{x}^{q} K_{l-1}(t, x-z ; \tau, \xi)\right| .
\end{gathered}
$$

In view of the boundedness of $\partial_{x}^{q} a_{1, k}^{l j}(t ; x),|q|_{+} \leq \alpha_{*}$, and estimate (2.1), for all $\{q, r\} \in \mathbb{Z}_{+}^{n},|q|_{+} \leq \alpha_{*},\{x, \eta, \xi\} \in \mathbb{R}^{n}, t \in(\tau ; T]$, and $\tau \in[0 ; T)$, we have

$$
\begin{aligned}
& \left|\partial_{\xi}^{r} \partial_{x}^{q} K_{1}(t, x ; \tau, \eta+\xi)\right| \leq m \sum_{|k|_{+} \leq p_{1}} \sum_{\left|q_{1}\right|_{+} \leq|q|_{+}} C_{q}^{q_{1}}\left|\partial_{x}^{q_{1}} A_{1, k}(t ; x)\right| \\
& \times\left|\partial_{(x-\eta-\xi)}^{k+r+q-q_{1}} G(t, \tau ; x-\eta-\xi)\right| \leq c_{r, q}(t-\tau)^{-\frac{n+p_{1}+\gamma+|r+q|_{+}}{h}} e^{-\delta\left(\frac{\|x-\eta-\xi\|}{(t-\tau)^{\alpha}}\right)^{\frac{1}{1-\alpha}}} \\
& \left|\partial_{x}^{q} K_{1}(t, x ; \tau, x-\xi)\right|=\left|\partial_{x}^{q}\left(\sum_{|k|_{+} \leq p_{1}} A_{1, k}(t ; x) \partial_{x}^{k} G(t, \tau ; \xi)\right)\right| \leq m\left|\partial_{x}^{q} A_{1,0}(t ; x)\right| \\
& \times|G(t, \tau ; \xi)| \leq \widehat{c}_{q}(t-\tau)^{-\frac{n+\gamma}{h}} e^{-\delta\left(\frac{\|\xi\|}{(t-\tau)^{\alpha}}\right)^{\frac{1}{1-\alpha}}} \leq c_{q}(t-\tau)^{-\frac{n+p_{1}+\gamma}{h}} e^{-\delta\left(\frac{\|\xi\|}{(t-\tau)^{\alpha}}\right)^{\frac{1}{1-\alpha}}}
\end{aligned}
$$


We now estimate the expression $\left|\partial_{\xi}^{r} K_{l}(t, \eta+\xi ; \tau, \xi)\right|$. Since

$$
\partial_{\xi}^{r} K_{1}(t, \eta+\xi ; \tau, \xi)=\sum_{|k|_{+\leq p_{1}}} \partial_{\xi}^{r} A_{1, k}(t ; \eta+\xi) \partial_{\eta}^{k} G(t, \tau ; \eta),(t, x ; \tau, \xi) \in \Pi_{T}^{2},
$$

we have, according to condition (A), that the iterated kernels $K_{l}(t, \eta+\xi ; \tau, \xi)$ are differentiable with respect to the variable $\xi$ only to the order $\alpha_{*}$. This fact and (3.2) imply that $\partial_{x}^{q} K_{l}(t, x ; \tau, \xi),|q|_{*} \leq \alpha_{*}$, is also a function differentiable with respect to $\xi$ only to this order $\alpha_{*}$.

Representation (3.5) and estimate (2.1) yield

$$
\left|\partial_{\xi}^{r} K_{1}(t, \eta+\xi ; \tau, \xi)\right| \leq c_{1, r}(t-\tau)^{-\frac{n+p_{1}+\gamma}{h}} e^{-\delta\left(\frac{\|\eta\|}{(t-\tau)^{\alpha}}\right)^{\frac{1}{1-\alpha}} .}
$$

We note that

$$
\partial_{\xi}^{r} K_{2}(t, \eta+\xi ; \tau, \xi)=\partial_{\xi}^{r}\left(\int_{\tau}^{t} d \beta \int_{\mathbb{R}^{n}} K_{1}(t, \eta+\xi ; \beta, y) K_{1}(\beta, y ; \tau, \xi) d y\right) .
$$

Let us change the variable of integration in the last integral by the formula $y=z+\xi$. In view of estimates (3.6) and (2.9) and the equalities

$$
\int_{\tau}^{t}((t-\beta)(\beta-\tau))^{\alpha_{0}-1} d \beta=(t-\tau)^{2 \alpha_{0}-1} B\left(\alpha_{0}, \alpha_{0}\right)
$$

and

we get

$$
\partial_{\xi}^{r} K_{1}(t, \eta+\xi ; \tau, z+\xi)=\left.\partial_{\zeta}^{r} K_{1}(t,(\eta-z)+\zeta ; \tau, \zeta)\right|_{\zeta=z+\xi},
$$

$$
\begin{aligned}
& \left|\partial_{\xi}^{r} K_{2}(t, \eta+\xi ; \tau, \xi)\right| \leq m \sum_{\left|r_{1}\right|+\leq|r|_{+}} C_{r}^{r_{1}} \int_{\tau}^{t} d \beta \int_{\mathbb{R}^{n}}\left|\partial_{\xi}^{r_{1}} K_{1}(t, \eta+\xi ; \beta, z+\xi)\right| \\
& \quad \times\left|\partial_{\xi}^{r-r_{1}} K_{1}(\beta, z+\xi ; \tau, \xi)\right| d z \leq m \sum_{\left|r_{1}\right|_{+\leq|r|_{+}} C_{r}^{r_{1}} c_{1, r_{1}} c_{1,\left(r-r_{1}\right)}}^{t} \\
& \quad \times \int_{\tau}^{t}((t-\beta)(\beta-\tau))^{-\frac{n+p_{1}+\gamma}{h}} \int_{\mathbb{R}^{n}} e^{-\delta\left(\left(\frac{\|n-z\| \|}{(t-\beta)^{\alpha}}\right)^{\frac{1}{1-\alpha}}+\left(\frac{\|z\|}{(\beta-\tau)^{\alpha}}\right)^{\frac{1}{1-\alpha}}\right)} d z d \beta \\
& \leq c_{2, r}(\varepsilon) B\left(\alpha_{0}, \alpha_{0}\right)(t-\tau)^{\alpha_{0}-\frac{n+p_{1}+\gamma}{h}} e^{-\delta(1-\varepsilon)\left(\frac{\|n\|}{(t-\tau)^{\alpha}}\right)^{\frac{1}{1-\alpha}}, \varepsilon \in(0 ; 1) .}
\end{aligned}
$$

By reasoning analogously step by step, we arrive at the inequality

$$
\left|\partial_{\xi}^{r} K_{l}(t, \eta+\xi ; \tau, \xi)\right| \leq c_{l, r}(\varepsilon)\left(\prod_{j=1}^{l-1} B\left(\alpha_{0}, j \alpha_{0}\right)\right)(t-\tau)^{(l-1) \alpha_{0}-\frac{n+p_{1}+\gamma}{h}}
$$




$$
\times e^{-\delta(1-(l-1) \varepsilon)\left(\frac{\|\eta\|}{(t-\tau)^{\alpha}}\right)^{\frac{1}{1-\alpha}}},
$$

which is satisfied for all $\{\eta, \xi\} \subset \mathbb{R}^{n},|r|_{+} \leq \alpha_{*}, 0 \leq \tau<t \leq T, \varepsilon \in(0 ; 1)$, and $l \in \mathbb{N} \backslash\{1\}$ and, hence, until the existence of such number $l_{*}$, for which

$$
\left|\partial_{\xi}^{r} K_{l_{*}}(t, \eta+\xi ; \tau, \xi)\right| \leq c_{l_{*}, r}(\varepsilon)\left(\prod_{j=1}^{l_{*}-1} B\left(\alpha_{0}, j \alpha_{0}\right)\right) e^{-\delta\left(1-\left(l_{*}-1\right) \varepsilon\right)\left(\frac{\|\eta\|}{(t-\tau)^{\alpha}}\right)^{\frac{1}{1-\alpha}}}
$$

(here, the quantities $c_{l, r}(\varepsilon)>0$ do not depend on the variables $t, \tau, \eta$, and $\xi$, which vary in the above-indicated way).

Since

$$
\begin{aligned}
\partial_{\xi}^{r} \partial_{x}^{q} K_{l}(t, x ; \tau, \eta+\xi) & =\left.\partial_{\zeta}^{r} \partial_{x}^{q} K_{l}(t, x ; \tau, \zeta)\right|_{\zeta=\eta+\xi}, \\
\partial_{\xi}^{r} \partial_{x}^{q} K_{l}(t, x-z ; \tau, \xi) & =\left.\partial_{\xi}^{r} \partial_{y}^{q} K_{l}(t, y ; \tau, \xi)\right|_{y=x-z},
\end{aligned}
$$

the expressions $\partial_{\xi}^{r} \partial_{x}^{q} K_{l}(t, x ; \tau, \eta+\xi), \partial_{\xi}^{r} \partial_{x}^{q} K_{l}(t, x-z ; \tau, \xi)$ and $\partial_{\xi}^{r} \partial_{x}^{q} K_{l}(t, x ; \tau, \xi)$ are of the same type. Therefore, with regard for representation (3.2) and estimates (3.3), (3.4), (3.8), and (2.9), we have

$$
\begin{aligned}
& \left|\partial_{\xi}^{r} \partial_{x}^{q} K_{2}(t, x ; \tau, \xi)\right| \leq m 2^{|r+q|_{+}}\left(\sum_{\left|r_{1}\right|_{+} \leq|r|_{+}} c_{r_{1}, q} c_{1,\left(r-r_{1}\right)} \int_{\tau}^{t_{1}}(t-\beta)^{-\frac{n+p_{1}+\gamma+\left|r_{1}+q\right|_{+}}{h}}\right. \\
& \times(\beta-\tau)^{-\frac{n+p_{1}+\gamma}{h}} \int_{\mathbb{R}^{n}} e^{-\delta\left(\left(\frac{\|x-\eta-\xi\|}{(t-\beta)^{\alpha}}\right)^{\frac{1}{1-\alpha}}+\left(\frac{\|n\|}{(\beta-\tau)^{\alpha}}\right)^{\frac{1}{1-\alpha}}\right)} d \eta d \beta \\
& +\sum_{\left|q_{1}\right|_{+} \leq|q|_{+}} c_{q_{1}} c_{r,\left(q-q_{1}\right)} \times \int_{t_{1}}^{t}(\beta-\tau)^{-\frac{n+p_{1}+\gamma+\left|r+q-q_{1}\right|_{+}}{h}}(t-\beta)^{-\frac{n+p_{1}+\gamma}{h}} \\
& \left.\int_{\mathbb{R}^{n}} e^{-\delta\left(\left(\frac{\|z\|}{(t-\beta)^{\alpha}}\right)^{\frac{1}{1-\alpha}}+\left(\frac{\|x-z-\xi\|}{(\beta-\tau)^{\alpha}}\right)^{\frac{1}{1-\alpha}}\right)} d z d \beta\right) \\
& \leq m 2^{|r+q|_{+}} c_{\varepsilon} e^{-\delta(1-\varepsilon)\left(\frac{\|x-\xi\|}{(t-\tau)^{\alpha}}\right)^{\frac{1}{1-\alpha}}(t-\tau)^{-\alpha n}} \sum_{\left|r_{1}\right|_{+} \leq|r|_{+}} c_{r_{1}, q} c_{1,\left(r-r_{1}\right)} \\
& \times \int_{\tau}^{t_{1}}(t-\beta)^{\alpha n-\frac{n+p_{1}+\gamma+\left|r_{1}+q\right|_{+}}{h}}(\beta-\tau)^{\alpha_{0}-1} d \beta+\sum_{\left|q_{1}\right|_{+} \leq|q|_{+}} c_{q_{1}} c_{r,\left(q-q_{1}\right)} \\
& \left.\quad \times \int_{t_{1}}^{t}(t-\beta)^{\alpha_{0}-1}(\beta-\tau)^{\alpha n-\frac{n+p_{1}+\gamma+\left|r+q-q_{1}\right|_{+}}{h}} d \beta\right),|r|_{+} \leq \alpha_{*},|q|_{+} \leq \alpha_{*}, \varepsilon \in(0 ; 1) .
\end{aligned}
$$


In view of the estimates

$$
\begin{aligned}
\int_{\tau}^{t_{1}}(t-\beta)^{\alpha n-\frac{n+p_{1}+\gamma+\left|r_{1}+q\right|_{+}}{h}}(\beta-\tau)^{\alpha_{0}-1} d \beta & \\
& \leq 2^{\frac{\left|r_{1}+q\right|_{+}}{h}}(t-\tau)^{2 \alpha_{0}-\left(1+\frac{\left|r_{1}+q\right|_{+}}{h}\right)} B\left(\alpha_{0}, \alpha_{0}\right)
\end{aligned}
$$

and

$$
\begin{array}{r}
\int_{t_{1}}^{t}(t-\beta)^{\alpha_{0}-1}(\beta-\tau)^{\alpha n-\frac{n+p_{1}+\gamma+\left|r+q-q_{1}\right|_{+}}{h}} d \beta \\
\leq 2^{\frac{\left|r+q-q_{1}\right|_{+}}{h}}(t-\tau)^{2 \alpha_{0}-\left(1+\frac{\left|r+q-q_{1}\right|_{+}}{h}\right)} B\left(\alpha_{0}, \alpha_{0}\right),
\end{array}
$$

we get the inequality

$$
\left|\partial_{\xi}^{r} \partial_{x}^{q} K_{2}(t, x ; \tau, \xi)\right| \leq c_{2, \xi}^{r, q}(t-\tau)^{2 \alpha_{0}-\left(1+\alpha n+\frac{|r+q|_{+}}{h}\right)} B\left(\alpha_{0}, \alpha_{0}\right) e^{-\delta(1-\varepsilon)\left(\frac{\|x-\xi\|}{(t-\tau)^{\alpha}}\right)^{\frac{1}{1-\alpha}}} .
$$

By continuing stepwise the process of estimation, we obtain

$$
\begin{aligned}
\left|\partial_{\xi}^{r} \partial_{x}^{q} K_{l}(t, x ; \tau, \xi)\right| & \leq c_{l, \varepsilon}^{r, q}(t-\tau)^{l \alpha_{0}-\left(1+\alpha n+\frac{|r+q|_{+}}{h}\right)} \\
& \leq e^{-\delta(1-(l-1) \varepsilon)\left(\frac{\|x-\xi\|}{(t-\tau)^{\alpha}}\right)^{\frac{1}{1-\alpha}}}\left(\prod_{j=1}^{l-1} B\left(\alpha_{0}, j \alpha_{0}\right)\right),
\end{aligned}
$$

for all $|r|_{+} \leq \alpha_{*},|q|_{+} \leq \alpha_{*},\{x, \xi\} \subset \mathbb{R}^{n}, 0 \leq \tau<t \leq T, \varepsilon \in(0 ; 1)$, and $l \in \mathbb{N} \backslash\{1\}$.

Let us pass to the estimation of the expression $\left|\partial_{\xi}^{r} \partial_{x}^{q} K_{l}(t, x ; \tau, \xi)\right|$, which will be suitable for the establishment of the differentiability of the matrix function $\Phi$ with respect to the spatial variables. Directly from (3.10), we arrive at the existence of a number $l^{*}$ such that

$$
\left|\partial_{\xi}^{r} \partial_{x}^{q} K_{l^{*}}(t, x ; \tau, \xi)\right| \leq c_{l^{*}, \varepsilon}^{r, q} e^{-\delta\left(1-\left(l^{*}-1\right) \varepsilon\right)\left(\frac{\|x-\xi\|}{(t-\tau)^{\alpha}}\right)^{\frac{1}{1-\alpha}}}\left(\prod_{j=1}^{l^{*}-1} B\left(\alpha_{0}, j \alpha_{0}\right)\right) .
$$

Let us set $l_{+}:=\max \left\{l_{*}, l^{*}\right\}, l_{-}:=\min \left\{l_{*}, l^{*}\right\}$, where $l_{*}$ is the corresponding number from (3.9), $\varepsilon:=\frac{1}{r_{*} l_{+}}, \delta_{*}:=\delta\left(1-\frac{1}{r_{*}}\right), r_{*}>2, T_{0}:=\max \{1, T\}$, and

$$
c_{*}^{0}:=\max _{l \in \mathbb{N}_{l_{+}} \backslash\{1\}}\left\{c_{1, r}, c_{l, r}(\varepsilon)\left(\prod_{j=1}^{l-1} B\left(\alpha_{0}, j \alpha_{0}\right)\right), c_{r, q}, c_{l, \varepsilon}^{r, q}\left(\prod_{j=1}^{l-1} B\left(\alpha_{0}, j \alpha_{0}\right)\right)\right\},
$$


$c_{*}:=c_{*}^{0}\left(T_{0}\right)^{l_{+}-l_{-}}$. Then (3.8) and (3.10) imply that, for all $\{x, \xi, \eta\} \subset \mathbb{R}^{n}, 0 \leq \tau<$ $t \leq T,|r|_{+} \leq \alpha_{*}$, and $|q|_{+} \leq \alpha_{*}$,

$$
\begin{gathered}
\left|\partial_{\xi}^{r} \partial_{x}^{q} K_{l_{+}}(t, x ; \tau, \xi)\right| \leq c_{*} e^{-\delta_{*}\left(\frac{\|x-\xi\|}{(t-\tau)^{\alpha}}\right)^{\frac{1}{1-\alpha}}}, \\
\left|\partial_{\xi}^{r} K_{l_{+}}(t, \eta+\xi ; \tau, \xi)\right| \leq c_{*} e^{-\delta_{*}\left(\frac{\|\eta\|}{(t-\tau)^{\alpha}}\right)^{\frac{1}{1-\alpha}} .}
\end{gathered}
$$

In view of this result, estimate (2.8), the equality

$$
\int_{\mathbb{R}^{n}} e^{-\delta_{0}\left(\frac{\|x-y\|}{(t-\beta)^{\alpha}}\right)^{\frac{1}{1-\alpha}}} \frac{d y}{(t-\beta)^{\alpha n}}=\int_{\mathbb{R}^{n}} e^{-\delta_{0}\|z\| \frac{1}{1-\alpha}} d z=: \widehat{E}<+\infty,
$$

representation (3.2), and inequalities (3.3) and (3.4), we obtain

$$
\begin{aligned}
& \left|\partial_{\xi}^{r} K_{l_{+}+1}(t, \eta+\xi ; \tau, \xi)\right| \leq \sum_{\left|r_{1}\right|_{+} \leq|r|_{+}} C_{r}^{r_{1}} \int_{\tau}^{t} d \beta \int_{\mathbb{R}^{n}} \mid \partial_{\xi}^{r_{1}} K_{1}(t, \eta+\xi ; \beta, z+\xi) \partial_{\xi}^{r-r_{1}} \\
& \times K_{l_{+}}(\beta, z+\xi ; \tau, \xi) \mid d z \leq m c_{*}^{2}\left(\sum_{\left|r_{1}\right|_{+} \leq|r|_{+}} C_{r}^{r_{1}}\right) \int_{\tau}^{t}(t-\beta)^{\alpha_{0}-1} \\
& \times \int_{\mathbb{R}^{n}} e^{-\delta_{*}\left(\left(\frac{\|\eta-z\| \|}{(t-\beta)^{\alpha}}\right)^{\frac{1}{1-\alpha}}+\left(\frac{\|z\|}{(\beta-\tau)^{\alpha}}\right)^{\frac{1}{1-\alpha}}\right)} e^{-\frac{\delta}{r_{*}}\left(\frac{\|\eta-z\| \|}{(t-\beta)^{\alpha}}\right)^{\frac{1}{1-\alpha}}} \frac{d z}{(t-\beta)^{n \alpha}} d \beta \\
& \leq m c_{r}^{0} \widehat{E} c_{*}^{2} B\left(\alpha_{0}, 1\right)(t-\tau)^{\alpha_{0}} e^{-\delta_{*}\left(\frac{\|\eta\|}{(t-\tau)^{\alpha}}\right)^{\frac{1}{1-\alpha}}}, c_{r}^{0}:=\sum_{\left|r_{1}\right|_{+} \leq|r|_{+}} C_{r}^{r_{1}} ; \\
& \left|\partial_{\xi}^{r} \partial_{x}^{q} K_{l_{+}+1}(t, x ; \tau, \xi)\right| \leq \sum_{\left|r_{1}\right|_{+} \leq|r|_{+}} C_{r}^{r_{1}} \int_{\tau}^{t_{1}} d \beta \int_{\mathbb{R}^{n}} \mid \partial_{\xi}^{r_{1}} \partial_{x}^{q} K_{1}(t, x ; \beta, \eta+\xi) \\
& \times \partial_{\xi}^{r-r_{1}} K_{l_{+}}(\beta, \eta+\xi ; \tau, \xi)\left|d \eta+\sum_{\left.\left|q_{1}\right|\right|_{+} \leq|q|_{+}} C_{q}^{q_{1}} \int_{t_{1}}^{t} d \beta \int_{\mathbb{R}^{n}}\right| \partial_{x}^{q_{1}} K_{1}(t, x ; \beta, x-z) \\
& \left.\times \partial_{\xi}^{r} \partial_{x}^{q-q_{1}} K_{l_{+}}(\beta, x-z ; \tau, \xi) \mid d z\right) \leq m c_{*}^{2}\left(\sum_{\left|r_{1}\right|_{+} \leq|r|_{+}} C_{r}^{r_{1}} \int_{\tau}^{t_{1}}(t-\beta)^{\alpha_{0}-\left(1+\frac{\left|r_{1}+q\right|_{+}}{h}\right)}\right. \\
& \times \int_{\mathbb{R}^{n}} e^{-\delta_{*}\left(\left(\frac{\|x-\eta-\xi\|}{(t-\beta)^{\alpha}}\right)^{\frac{1}{1-\alpha}}+\left(\frac{\|\eta\|}{(\beta-\tau)^{\alpha}}\right)^{\frac{1}{1-\alpha}}\right)} e^{-\frac{\delta}{r_{*}}\left(\frac{\|x-\eta-\xi\|}{(t-\beta)^{\alpha}}\right)^{\frac{1}{1-\alpha}}} \frac{d \eta}{(t-\beta)^{\alpha n}} d \beta
\end{aligned}
$$




$$
\begin{aligned}
& +\sum_{\left|q_{1}\right|_{+} \leq|q|_{+}} C_{q}^{q_{1}} \int_{t_{1}}^{t}(t-\beta)^{\alpha_{0}-1} \int_{\mathbb{R}^{n}} e^{-\delta_{*}\left(\left(\frac{\|z\|}{(t-\beta)^{\alpha}}\right)^{\frac{1}{1-\alpha}}+\left(\frac{\|x-z-\xi\|}{(\beta-\tau)^{\alpha}}\right)^{\frac{1}{1-\alpha}}\right)} \\
& \left.\times e^{-\frac{\delta}{r *}\left(\frac{\|z\|}{(t-\beta)^{\alpha}}\right)^{\frac{1}{1-\alpha}}} \frac{d z}{(t-\beta)^{\alpha n}} d \beta\right) \leq m c_{*}^{2} \widehat{E} e^{-\delta_{*}\left(\frac{\|x-\xi\|}{(t-\tau)^{\alpha}}\right)^{\frac{1}{1-\alpha}}} \int_{\tau}^{t}(t-\beta)^{\alpha_{0}-1} d \beta \\
& \times\left(\left(\sum_{\left|r_{1}\right|_{+} \leq|r|_{+}} C_{r}^{r_{1}}\left(t-t_{1}\right)^{-\frac{\left|r_{1}+q\right|_{+}}{h}}\right)+c_{q}\right) \leq m c_{*}^{2} \widehat{E} e^{-\delta_{*}\left(\frac{\|x-\xi\|}{(t-\tau)^{\alpha}}\right)^{\frac{1}{1-\alpha}}}(t-\tau)^{\alpha_{0}} \\
& \times B\left(\alpha_{0}, 1\right)\left(\left(2^{\frac{|r+q|_{+}}{h}} \sum_{\left|r_{1}\right|_{+} \leq|r|_{+}} C_{r}^{r_{1}}(t-\tau)^{-\frac{\left|r_{1}+q\right|_{+}}{h}}\right)+c_{q}\right) \\
& \leq m c_{r, q}^{0} c_{*}^{2} \widehat{E}\left(2 T_{0}\right)^{\frac{|r+q|_{+}}{h}} B\left(\alpha_{0}, 1\right)(t-\tau)^{\alpha_{0}-\frac{|r+q|_{+}}{h}} e^{-\delta_{*}\left(\frac{\|x-\xi\|}{(t-\tau)^{\alpha}}\right)^{\frac{1}{1-\alpha}}}, c_{r, q}^{0}:=c_{r}+c_{q} .
\end{aligned}
$$

Applying the method of induction, we can verify firstly the validity of the estimate

$$
\begin{aligned}
& \quad\left|\partial_{\xi}^{r} K_{l_{+}+l}(t, \eta+\xi ; \tau, \xi)\right| \\
& \quad \leq c_{*}\left(m c_{r}^{0} c_{*} \widehat{E}(t-\tau)^{\alpha_{0}}\right)^{l} e^{-\delta_{*}\left(\frac{\|\eta\|}{(t-\tau)^{\alpha}}\right)^{\frac{1}{1-\alpha}}}\left(\prod_{j=1}^{l-1} B\left(\alpha_{0}, 1+j \alpha_{0}\right)\right),
\end{aligned}
$$

and, hence, the estimate

$$
\begin{aligned}
\left|\partial_{\xi}^{r} \partial_{x}^{q} K_{l_{+}+l}(t, x ; \tau, \xi)\right| \leq & c_{*}\left(m c_{r, q}^{0} c_{*} \widehat{E}\left(2 T_{0}\right)^{\frac{|r+q|_{+}}{h}}\right)^{l}(t-\tau)^{l \alpha_{0}-\frac{|r+q|_{+}}{h}} \\
& \times e^{-\delta_{*}\left(\frac{\|x-\xi\|}{(t-\tau)^{\alpha}}\right)^{\frac{1}{1-\alpha}}}\left(\prod_{j=1}^{l-1} B\left(\alpha_{0}, 1+j \alpha_{0}\right)\right),
\end{aligned}
$$

for $|r|_{+} \leq \alpha_{*},|q|_{+} \leq \alpha_{*},(t, x ; \tau, \xi) \in \Pi_{T}^{2}$ and $l \in \mathbb{N} \backslash\{1\}$.

The following propositions hold true.

Lemma 1. The matrix function $\Phi(t, x ; \tau, \xi)$ on the set $\Pi_{T}^{2}$ is a function differentiable with respect to each of the spatial variables $x$ and $\xi$ to the order $\alpha_{*}$ inclusively, and their derivatives satisfy the following estimates:

$$
\begin{gathered}
\left|\partial_{\xi}^{r} \partial_{x}^{q} \Phi(t, x ; \tau, \xi)\right| \leq c_{1}(t-\tau)^{\alpha_{0}-\left(1+\alpha n+\frac{|r+q|_{+}}{h}\right)} e^{-\delta_{*}\left(\frac{\|x-\xi\|}{(t-\tau)^{\alpha}}\right)^{\frac{1}{1-\alpha}}}, \\
\left|\partial_{\xi}^{r} \Phi(t, \eta+\xi ; \tau, \xi)\right| \leq c_{2}(t-\tau)^{\alpha_{0}-(1+\alpha n)} e^{-\delta_{*}\left(\frac{\|n\| \|}{(t-\tau)^{\alpha}}\right)^{\frac{1}{1-\alpha}}},\{\eta, \xi\} \subset \mathbb{R}^{n}
\end{gathered}
$$

(here, the estimating constants $c_{1}, c_{2}$, and $\delta_{*}$ are independent of $t, \tau, x, \xi, \eta$ ). 
Proof. In any way, let us fix a point $\left(x_{0} ; \xi_{0}\right)$ from $\mathbb{R}^{2 n}$, and let us consider a ball $\mathbb{K}_{\left(x_{0} ; \xi_{0}\right)}^{\delta}$ with radius $\delta>0$, which is centered at the point $\left(x_{0} ; \xi_{0}\right)$, in this space. Then, in view of structure (2.4) of the function $\Phi$ and the differentiability of the iterated kernels $K_{l}$ with respect to spatial variables on $\mathbb{R}^{2 n}$ to the order $\alpha_{*}$ inclusively, we can conclude that, in order to prove the differentiability of the matrix function $\Phi$ at the point $\left(x_{0} ; \xi_{0}\right)$ to the indicated order, it is necessary only to prove the uniform convergence of the formally differentiated series (2.4) in the variables $x$ and $\xi$ on the set $\mathbb{K}_{\left(x_{0} ; \xi_{0}\right)}^{\delta}, \delta>0$ (at every fixed $t$ and $\tau, 0 \leq \tau<t \leq T$ ):

$$
\sum_{l=1}^{\infty} \partial_{\xi}^{r} \partial_{x}^{q} K_{l}(t, x ; \tau, \xi), \quad|r|_{+} \leq \alpha_{*},|q|_{+} \leq \alpha_{*} .
$$

Directly from estimates (3.10) and (3.12) and the equality

$$
\prod_{j=0}^{l-1} B\left(\alpha_{0}, 1+j \alpha_{0}\right)=\frac{\left(\Gamma\left(\alpha_{0}\right)\right)^{l}}{\Gamma\left(1+l \alpha_{0}\right)},
$$

where $\Gamma(\cdot)$ is the Euler gamma-function, for $\{r, q\} \subset \mathbb{Z}_{+}^{n},|r|_{+} \leq \alpha_{*},|q|_{+} \leq \alpha_{*}$, and $(t, x ; \tau, \xi) \in \Pi_{T}^{2}$, we have

$$
\begin{aligned}
& \left|\sum_{l=1}^{\infty} \partial_{\xi}^{r} \partial_{x}^{q} K_{l}(t, x ; \tau, \xi)\right| \leq \sum_{l=1}^{l_{+}}\left|\partial_{\xi}^{r} \partial_{x}^{q} K_{l}(t, x ; \tau, \xi)\right|+\sum_{l=l_{+}+1}^{\infty}\left|\partial_{\xi}^{r} \partial_{x}^{q} K_{l}(t, x ; \tau, \xi)\right| \\
& \leq c_{*}\left(\sum_{l=1}^{l_{+}}(t-\tau)^{l \alpha_{0}-\left(1+\alpha n+\frac{|r+q|_{+}}{h}\right)}\right. \\
& \quad+\sum_{l=1}^{\infty}\left(m c_{r, q}^{0} c_{*} \widehat{E}\left(2 T_{0}\right)^{\frac{|r+q|_{+}}{h}}\right)^{l}(t-\tau)^{l \alpha_{0}-\frac{|r+q|_{+}}{h}} \\
& \left.\quad \times\left(\prod_{j=1}^{l-1} B\left(\alpha_{0}, 1+j \alpha_{0}\right)\right)\right) e^{-\delta_{*}\left(\frac{\|x-\xi\|}{(t-\tau)^{\alpha}}\right)^{\frac{1}{1-\alpha}}} \leq c_{1}(t-\tau)^{\alpha_{0}-\left(1+\alpha n+\frac{|r+q|_{+}}{h}\right)} \\
& \quad \times e^{-\delta_{*}\left(\frac{\|x-\xi\|}{(t-\tau)^{\alpha}}\right)^{\frac{1}{1-\alpha}} .}
\end{aligned}
$$

From whence, we get the uniform convergence of series (3.15) in $x$ and $\xi$ and, hence, the validity of estimates (3.13).

Due to the corresponding estimates (3.8) and (3.11), we can verify analogously the validity of estimate (3.14).

The lemma is proved.

Lemma 2. The volumetric potential $W(t, x ; \tau, \xi)$ on the set $\Pi_{T}^{2}$ is a function differentiable with respect to each of the spatial variables $x$ and $\xi$ to, respectively, the 
orders $\alpha_{*}+p_{1}$ and $\alpha_{*}$ inclusively. In this case,

$$
\begin{gathered}
\partial_{\xi}^{r} \partial_{x}^{q} W(t, x ; \tau, \xi)=\sum_{l=0}^{r} C_{r}^{l} \int_{\tau}^{t_{1}} d \beta \int_{\mathbb{R}^{n}} \partial_{\xi}^{l} \partial_{x}^{q} G(t, \beta ; x-y-\xi) \partial_{\xi}^{r-l} \Phi(\beta, y+\xi ; \tau, \xi) d y \\
+\int_{t_{1}}^{t} d \beta \int_{\mathbb{R}^{n}} \partial_{x}^{q} G(t, \beta ; x-y) \partial_{\xi}^{r} \Phi(\beta, y ; \tau, \xi) d y,|q|_{+} \leq p_{1},|r|_{+} \leq \alpha_{*}, \quad \text { (3.16) } \\
\partial_{\xi}^{r} \partial_{x}^{q} W(t, x ; \tau, \xi)= \\
\sum_{l=0}^{r} C_{r}^{l} \int_{\tau}^{t_{1}} d \beta \int_{\mathbb{R}^{n}} \partial_{\xi}^{l} \partial_{x}^{q} G(t, \beta ; x-y-\xi) \partial_{\xi}^{r-l} \Phi(\beta, y+\xi ; \tau, \xi) d y \\
+\int_{t_{1}}^{t} d \beta \int_{\mathbb{R}^{n}} \partial_{\eta}^{k} G(t, \beta ; \eta) \partial_{\xi}^{r} \partial_{x}^{q-k} \Phi(\beta, x-\eta ; \tau, \xi) d \eta,|r|_{+} \leq \alpha_{*} \\
|k|_{+}=p_{1}, p_{1}<|q|_{+} \leq \alpha_{*}+p_{1} .
\end{gathered}
$$

Proof. For $|q|_{+} \leq p_{1}$ and $|r|_{+} \leq \alpha_{*}$, we use the representation

$$
\begin{aligned}
W(t, x ; \tau, \xi)= & \int_{\tau}^{t_{1}} d \beta \int_{\mathbb{R}^{n}} G(t, \beta ; x-y-\xi) \Phi(\beta, y+\xi ; \tau, \xi) d y \\
& +\int_{t_{1}}^{t} d \beta \int_{\mathbb{R}^{n}} G(t, \beta ; x-y) \Phi(\beta, y ; \tau, \xi) d y .
\end{aligned}
$$

From whence, by the formal differentiation under the sign of integral, we obtain equality (3.16). Hence, in order to substantiate the validity of equality (3.16), it is sufficient to prove the uniform convergence of the following integrals in the variables $x$ and $\xi$ on $\mathbb{R}^{2 n}$ :

$$
\begin{aligned}
& d_{1}^{r, l, q}\left(t_{1}, x ; \tau, \xi\right):=\int_{\tau}^{t_{1}} d \beta \int_{\mathbb{R}^{n}}\left|\partial_{\xi}^{l} \partial_{x}^{q} G(t, \beta ; x-y-\xi)\right|\left|\partial_{\xi}^{r-l} \Phi(\beta, y+\xi ; \tau, \xi)\right| d y, \\
& |l|_{+} \leq|r|_{+} ; d_{2}^{r, q}\left(t, x ; t_{1}, \xi\right):=\int_{t_{1}}^{t} d \beta \int_{\mathbb{R}^{n}}\left|\partial_{x}^{q} G(t, \beta ; x-y) \| \partial_{\xi}^{r} \Phi(\beta, y ; \tau, \xi)\right| d y .
\end{aligned}
$$


This convergence becomes obvious, if we take condition (A) and the following estimates into account for $\{x, \xi\} \subset \mathbb{R}^{n}$ and $0 \leq \tau<t \leq T$ :

$$
\begin{aligned}
d_{1}^{r, l, q}\left(t_{1}, x ; \tau, \xi\right) \leq & c c_{2} \widehat{E} e^{-\delta_{*}\left(\frac{\|x-\xi\|}{(t-\tau)^{\alpha}}\right)^{\frac{1}{1-\alpha}}}\left(t-t_{1}\right)^{-\frac{n+\gamma+|l+q|_{+}}{h}} \\
& \times \int_{\tau}^{t_{1}}(\beta-\tau)^{\alpha_{0}-1} d \beta,|l|_{+} \leq|r|_{+}
\end{aligned}
$$

$$
\begin{aligned}
& d_{2}^{r, q}\left(t, x ; t_{1}, \xi\right) \\
& \leq c c_{1} \widehat{E} e^{-\delta_{*}\left(\frac{\|x-\xi\| \|}{(t-\tau)^{\alpha}}\right)^{\frac{1}{1-\alpha}}}\left(t_{1}-\tau\right)^{-\frac{n+p_{1}+\gamma+|r|_{+}}{h}} \int_{t_{1}}^{t}(t-\beta)^{\alpha_{0}-1+\frac{p_{1}-|q|_{+}}{h}} d \beta .
\end{aligned}
$$

These estimates follow directly from (2.1), (3.13), and (3.14).

We now prove the validity of formula (3.17). For this purpose, we fix any $k \in \mathbb{Z}_{+}^{n}$ such that $|k|_{+}=p_{1}$. Then, according to (3.16) for $p_{1}<|q|_{+} \leq \alpha_{*}+p_{1}$ and $|r|_{+} \leq$ $\alpha_{*}$, we have

$$
\begin{gathered}
\partial_{\xi}^{r} \partial_{x}^{q} W(t, x ; \tau, \xi)=\sum_{l=0}^{r} C_{r}^{l} \partial_{x}^{q-k} \int_{\tau}^{t_{1}} d \beta \int_{\mathbb{R}^{n}} \partial_{\xi}^{l} \partial_{x}^{k} G(t, \beta ; x-y-\xi) \partial_{\xi}^{r-l} \Phi(\beta, y+\xi ; \tau, \xi) \\
\times d y+\partial_{x}^{q-k} \int_{t_{1}}^{t} d \beta \int_{\mathbb{R}^{n}} \partial_{\eta}^{k} G(t, \beta ; \eta) \partial_{\xi}^{r} \Phi(\beta, x-\eta ; \tau, \xi) d \eta,(t, x ; \tau, \xi) \in \Pi_{T}^{2} .
\end{gathered}
$$

Hence, it remains to substantiate the possibility to introduce the operation $\partial_{x}^{q-k}$ under the signs of the corresponding integrals. In other words, we should prove the uniform convergence in $x$ and $\xi$ of the following integrals on $\mathbb{R}^{2 n}$ for $0 \leq \tau<t \leq T$ :

$$
\begin{gathered}
\int_{\tau}^{t_{1}} d \beta \int_{\mathbb{R}^{n}} \partial_{\xi}^{l} \partial_{x}^{q} G(t, \beta ; x-y-\xi) \Phi(\beta, y+\xi ; \tau, \xi) d y, \\
\int_{t_{1}}^{t} d \beta \int_{\mathbb{R}^{n}} \partial_{\eta}^{k} G(t, \beta ; \eta) \partial_{\xi}^{r} \partial_{x}^{q-k} \Phi(\beta, x-\eta ; \tau, \xi) d \eta .
\end{gathered}
$$

By reasoning similarly to the case of integrals (3.18) and using estimates (2.1), (3.13), and (3.14), we get the necessary convergence of the indicated integrals.

The lemma is proved.

The main result can be formulated as the following proposition. 
Theorem 1. Let system (1.1) satisfy conditions $(A)$ and (B). Then the corresponding function $Z(t, x ; \tau, \xi)$ defined by equality (2.2) is a function differentiable with respect to each of the spatial variables $x$ and $\xi$ on the set $\Pi_{T}^{2}$ to, respectively, the orders $\alpha_{*}+p_{1}$ and $\alpha_{*}$ inclusively, and

$$
\begin{gathered}
\exists \delta>0 \forall\{r, q\} \subset \mathbb{Z}_{+}^{n},|q|_{+} \leq \alpha_{*}+p_{1},|r|_{+} \leq \alpha_{*}, \exists c>0 \forall(t, x ; \tau, \xi) \in \Pi_{T}^{2}: \\
\left|\partial_{\xi}^{r} \partial_{x}^{q} Z(t, x ; \tau, \xi)\right| \leq c(t-\tau)^{-\frac{n+|r+q|_{+}+\gamma}{h}} e^{-\delta\left(\frac{\|x-\xi\|}{(t-\tau)^{\alpha}}\right)^{\frac{1}{1-\alpha}}} \\
\left|\partial_{\xi}^{k} Z(t, x+\xi ; \tau, \xi)\right| \leq c_{k}(t-\tau)^{\beta_{k}-\frac{n+\gamma}{h}} e^{-\delta_{1}\left(\frac{\|x\|}{(t-\tau)^{\alpha}}\right)^{\frac{1}{1-\alpha}}}
\end{gathered}
$$

where $|k|_{+} \leq \alpha_{*}, 0 \leq \tau<t \leq T,\{x, \xi\} \subset \mathbb{R}^{n}, \beta_{k}:=\left\{\begin{array}{ll}0, & k=0, \\ \alpha_{0}, & k \neq 0\end{array}\right.$ (here, the estimating constants are independent of $t, \tau, x$, and $\xi$ ).

Proof. With regard for structure (2.2) and the infinite differentiability of the function $G(t, \tau ; \xi)$ with respect to the variable $\xi$, the smoothness of the function $Z(t, x ; \tau, \xi)$ in the variables $x$ and $\xi$ becomes obvious directly from the assertion of Lemma 2.

Let $|q|_{+} \leq p_{1}$ and $|r|_{+} \leq \alpha_{*}$. Then, according to (3.16), we get

$\left|\partial_{\xi}^{r} \partial_{x}^{q} Z(t, x ; \tau, \xi)\right| \leq\left|\partial_{x-\xi}^{r+q} G(t, \tau ; x-\xi)\right|+\sum_{l=0}^{r} C_{r}^{l} \alpha_{1}^{r, l, q}\left(t_{1}, x ; \tau, \xi\right)+d_{2}^{r, q}\left(t, x ; t_{1}, \xi\right)$.

From whence, by using estimates (2.1), (3.19), and (3.20), we obtain assertion (3.21).

In a similar way, by using formula (3.17), we verify the validity of assertion (3.21) also for $p_{1}<|q|_{+} \leq \alpha_{*}$ and $|r|_{+} \leq \alpha_{*}$.

Then, according to estimates (2.1) and (3.14), we have

$$
\begin{aligned}
& Y_{k}(t, x ; \tau, \xi):=\left|\int_{\tau}^{t} d \beta \int_{\mathbb{R}^{n}} G(t, \beta ; x-\zeta) \partial_{\xi}^{k} \Phi(\beta, \zeta+\xi ; \tau, \xi) d \zeta\right| \\
& \leq c c_{2} \int_{\tau}^{t}(t-\beta)^{\alpha_{0}+\frac{p_{1}}{h}-1}(\beta-\tau)^{\alpha_{0}-1} \int_{\mathbb{R}^{n}} \exp \left\{-\delta_{0}\left\{\left(\frac{\|x-\zeta\|}{(t-\beta)^{\alpha}}\right)^{\frac{1}{1-\alpha}}\right.\right. \\
& \left.\left.\quad+\left(\frac{\|\zeta\|}{(\beta-\tau)^{\alpha}}\right)^{\frac{1}{1-\alpha}}\right\}\right\} \frac{d y d \beta}{\left((t-\beta)(\beta-\tau)^{\alpha n}\right.}, \delta_{0}:=\min \left\{\delta, \delta_{*}\right\},|k|_{+} \leq \alpha_{*} .
\end{aligned}
$$

Using estimate (2.9) and equality (3.7), we get

$$
Y_{k}(t, x ; \tau, \xi) \leq c_{\varepsilon}(t-\tau)^{\alpha_{0}-\frac{n+\gamma}{h}} e^{-\delta_{0}(1-\varepsilon)\left(\frac{\|x\|}{(t-\tau)^{\alpha}}\right)^{\frac{1}{1-\alpha}}}, \quad \varepsilon \in(0 ; 1),
$$


where $|k|_{+} \leq \alpha_{*}, 0 \leq \tau<t \leq T$ and $\{x, \xi\} \subset \mathbb{R}^{n}$. From whence, with regard for inequality (2.1) and the representation

$$
Z(t, x+\xi ; \tau, \xi)=G(t, \tau ; x)+\int_{\tau}^{t} d \beta \int_{\mathbb{R}^{n}} G(t, \beta ; x-\zeta) \Phi(\beta, \zeta+\xi ; \beta, \xi) d \zeta
$$

we arrive at estimate (3.22).

The theorem is proved.

\section{REFERENCES}

[1] A. Friedman, Partial Differential Equations of Parabolic Type. Englewood Cliffs: Prentice-Hall, 1964.

[2] I. Gelfand and G. Shilov, Generalized Functions. Vol. 3. Theory of Differential Equations. Boston, MA: Academic Press, 1967.

[3] I. Gelfand and G. Shilov, Generalized Functions. Vol. 2. Spaces of Fundamental and Generalized Functions. Boston, MA: Academic Press, 1968.

[4] U. Hou-sin, "On the definition of parabolicity of systems of equations with partial derivatives." Uspekhi Mat. Nauk, vol. 15, no. 6, pp. 157-161, 1960.

[5] I.M.Dovzhytska, "The Cauchy problem for Shilov-type parabolic systems with variable coefficients and nonnegative genus." Author's abstract of the Candidate thesis, Yu. Fed'kovych Chernivtsi National University, 2, Kotsubins'ky Str., 58012 Chernivtsi, Ukraine, 30 January 2015.

[6] V. Litovchenko, "The Cauchy problem for parabolic equations by Shilov." Siber. Mat. Zh., vol. 45, no. 4, pp. 809-821, 2004, doi: 10.1023/B:SIMJ.0000035831.63036.bb.

[7] V. Litovchenko and I. Dovzhytska, "The fundamental matrix of solutions of the Cauchy problem for a class of parabolic system of the Shilov type with variable coefficients." J. of Math. Sci., vol. 175, no. 4, pp. 450-476, 2011, doi: 10.1007/s10958-011-0356-0.

[8] V. Litovchenko and I. Dovzhytska, "Cauchy problem for a class of parabolic systems of Shilov type with variable coefficients." Cent. Eur. J. Math., vol. 10, no. 3, pp. 1084-1102, 2012, doi: 10.2478/s11533-012-0025-7.

[9] V. Litovchenko and I. Dovzhytska, "Stabilization of solutions of Shilov-type parabolic systems with nonnegative genus." Siber. Mat. Zh., vol. 55, no. 2, pp. 341-349, 2014, doi: 10.1134/S0037446614020104.

[10] Y. Zhitomirskii, "Cauchy problem for some types of systems of linear equations, which are parabolic by G.E. Shilov, with partial derivatives with continuous coefficients." Izv. AN SSSR. Ser. Mat., vol. 23, pp. 925-932, 1959.

Authors' addresses

\section{Litovchenko}

Yu. Fed'kovych Chernivtsi National University, 2, Kotsubins'ky Str., 58012 Chernivtsi, Ukraine

E-mail address: vladlit4@mail.ru

\section{G. Unguryan}

Yu. Fed'kovych Chernivtsi National University, 2, Kotsubins'ky Str., 58012 Chernivtsi, Ukraine

E-mail address: galuna_unguryan@ukr.net 